\title{
EDITORIAL
}

\section{Ventilation During Anaesthesia}

The ReALIZATION that respiratory acidosis due to retention of carbon dioxide durmg the depression of respiratory activity associated with anaesthesia may lead to important disturbances of function has resulted in a laudable interest in maintaining adequate ventilation in the anaesthetized patient. We have become awar?, however, that there may be a tendency for some anaesthetists to lose sight of the basic nature of the disturbance which does harm. and by being over-zealous in the elımination of carbon dioxide to produce, in fact, the very situation which they labour so hard to avoid.

It must be borne in mind that the disturbance which is damaging to the normal body functions is a shift in electrolyte balance and distribution. Such a disturbance will appear with equal readiness on the production of a relative acıdosis or alkalosis. Haldane (1) showed many years ago that the body can accommodate to the presence of high concentrations of carbon dioxide in the respired atmosphere, but that sudden change in this concentration would produce disturbances approaching catastrophic proportions. We have known emotionally mduced hyperventilation to produce tetanic convulsions and vomiting in an apparently healthy individual

We feel that perhaps too much emphasis is being placed on the hyperventilation of anaesthetized patients While it is undoubtedly true, as Dr. Cullen and his associates point out elsewhere in this Journal, that a volume of ventilation somewhat exceeding the normal is required to produce adequate exchange in these patients, we are alarmed when the production of a blood pH grossly on the alkaline side of normal is hailed as a triumph in the elimination of carbon dioxide. Let us now beware lest this become a hobby-horse which will prove as dangerous as its Trojan ancestor. We should undoubtedly be safer to think more in terms of maintaining a normal respiratory exchange in the individual patient than of trying to get rid of all possible carbon dioxide.

\section{REFERENCE}

1 Haldane, J S, and Priestley J G. Respuration Oxford-At the Clarendon Press (1935) 


\section{VENTILATION DURANT L'ANESTHÉSTE}

C'EST un fait que l'acidose ıspiratoire due à une rétention de gaz carbonique à la suite d'une diminution de l'activité respiratoire en relation avec l'anesthésie peut conduire à d'importants troubles fonctionnels; cette notion a fait naître un grand intérêt dans le maintien d'une ventilation adéquate chez le malade sous anesthésie. Toutefois, ll faut être avisé: ll y a tendance, chez certains anesthésistes, à perdre de vue le principe à respecter pour éviter les perturbations nuisibles; puis, en étant trop zélés pour faire éliminer le gaz carbonique, ces anesthésistes produisent, en fait, la situation qu'ils ont si laborieusement essayé d'éviter.

Il faut se rappeler que les perturbations qui deviennent nuisibles aux fonctions normales de l'organisme résident dans le déplacement des électrolytes et leur redistribution. Ces perturbations peuvent apparaître aussi promptement à l'occasion d'une acidose relative qu'à l'occasion d'une alcalose relative. Il y a quelques années, Haldane (1) a démontré que l'organisme peut supporter de hautes concentrations de gaz carbonique dans l'air inspiré, mais que le changement brusque de cette concentration peut produire des perturbations conduisant à des catastrophes. Nous avons été témoins d'une hyperventilation, engendrée par l'émotion, qui, chez un individu en bonne santé apparente, a provoqué des vomissements et des convulsions tétaniques

Nous avons l'impression qu'on insiste trop sur l'hyperventilation des malades sous anesthésie. Sans doute il est vrai, comme l'affirmarent, quelque part dans ce journal, le Docteur Cullen et ses associés, qu'il falut faire une ventilation d'un volume dépassant légèrement la normale pour assurer des échanges adéquats chez ces malades, mass nous devenons consternés quand on nous annonce, comme un triomphe sur l'élimination du gaz carbonique, un $\mathrm{pH}$ sanguin fortement alcalin Soyons bien avertis que ce cheval die bataille peut s'avérer aussi dangereux que celu de l'ancêtre Trojan Il serait sans doute plus prudent de penser à maintenir des échanges respiratorres normaux pour un malade en particulier que d'essayer de faire élıminer le plus de gaz carbonique possible. 